\title{
Synthesis and Characterisation of Functionalised-Graphene Oxide by Gamma-Ray Irradiation
}

\author{
Noraniza Ahmad Daud ${ }^{1}$, Buong Woei Chieng ${ }^{1,2}$, Nor Azowa Ibrahim ${ }^{1,2}$ and \\ Zainal Abidin Talib ${ }^{3}$ \\ ${ }^{1}$ Department of Chemistry, Faculty of Science, Universiti Putra Malaysia, \\ 43400 UPM Serdang, Selangor, Malaysia \\ ${ }^{2}$ Materials Processing and Technology Laboratory, Institute of Advanced \\ Technology, Universiti Putra Malaysia, 43400 UPM Serdang, Selangor, Malaysia \\ ${ }^{3}$ Department of Physics, Faculty of Science, Universiti Putra Malaysia, \\ 43400 UPM Serdang, Selangor, Malaysia \\ *Corresponding author: norazowa@upm.edu.my
}

Published online: 15 April 2017

To cite this article: Noraniza Ahmad Daud, Buong Woei Chieng, Nor Azowa Ibrahim and Zainal Abidin Talib. (2017). Synthesis and characterisation of functionalisedgraphene oxide by gamma-ray irradiation. Journal of Engineering Science, 13: 1-17, https://doi.org/10.21315/jes2017.13.1.

To link to this article: https://doi.org/10.21315/jes2017.13.1

\begin{abstract}
Gamma-ray irradiation technique was used to functionalise graphene oxide (GO) withvarious alkyl chain length alkylamine. Functionalisation of alkylchain onto the GO was confirmed by nuclear magnetic resonance $\left({ }^{l} H N M R\right)$, Fourier transform infrared (FTIR) and X-ray diffraction (XRD). FTIR of functionalised GO showed the appearance of significant peaks around 2850-2960 $\mathrm{cm}^{-1}\left(-\mathrm{CH}_{2}\right)$ which come from long alkylchain together with peak around $1450-1560 \mathrm{~cm}^{-1}$ indicating the formation of $\mathrm{C}-\mathrm{NH}-\mathrm{C} . \mathrm{XRD}$ showed an additional diffraction peak at lower $2 \theta$ angle, indicating that the intercalation of alkylamine was successful. The effects of various alkyl lengths functionalised-GO on morphological and thermal properties were investigated. Scanning electron microscopy (SEM) analysis showed an increase in surface roughness when the alkyl chain length increases. The addition of alkylchain on GO surfaces significantly improves the thermal stability of $G O$, suggesting their great potential for hydrophobic material in industry.
\end{abstract}

Keywords: Graphene oxide, functionalisation, alkylamine, gamma-irradiation

\section{INTRODUCTION}

Graphene has drawn a great deal of attention from scientific communities in recent years due to its excellent mechanical, thermal, optical and electronic properties. It's a new class of two-dimensional carbon nanostructure with a single 
layer of $\mathrm{sp}^{2}$ network of atom in honeycomb lattice. It can be produced by mechanical exfoliation of graphite, epitaxial growth, chemical vapour deposition (CVD), liquid-phase exfoliation ${ }^{1}$, solvent-thermal ${ }^{2}$ and the reduction of graphene oxide (GO). Among them, reduction of GO is the most economic method for synthesis of graphene which based on exfoliation of graphite into one atom thick sheets by oxidation, creating GO and subsequent reduction of GO into graphene sheet by reducing agents such as hydrazine, hydroquinone, vitamin $\mathrm{C}$, sodium borohydride, dimethylformamide, urea and so on. Reduced GO approach the highly desired properties of graphene such as electric conductivity to various degrees, but not completely.

On the other hand, GO is a graphene sheet with oxygen functional group on its edges and basal plane, as proposed by Lerf and coworkers. ${ }^{3}$ It is believed that carboxylic and carbonyl groups are at its edges, whereas phenol, hydroxyl, and epoxide groups are on its basal plane. ${ }^{4} \mathrm{GO}$ is an interesting derivative of carbon material which has sparked a tremendous attention from scientist communities in recent years due to its unique and amazing properties. It has been proposed uses as electronic devices, sensors, energy materials, hydrogen storage materials and also as a value added-filler in composite materials.

Brodie, in year 1859, first demonstrated the synthesis of GO by adding a portion of potassium chlorate to a slurry of graphite in fuming nitric acid. In year 1898, Staudenmaier improved on this protocol by using concentrated sulphuric acid as well as fuming nitric acid and adding the chlorate in multiple aliquots over the course of the reaction. In year 1958, Hummers reported the method most commonly used today, oxidising graphite by treatment with $\mathrm{KMnO}_{4}$ and $\mathrm{NaNO}_{3}$ in concentrated $\mathrm{H}_{2} \mathrm{SO}_{4}$. However, all three of these procedures involve the generation of the toxic or explosive gases $\mathrm{NO}_{2}, \mathrm{~N}_{2} \mathrm{O}_{4}$ and $\mathrm{ClO}_{2}$. Various synthesis methods are summarised in Table 1.

Table 1: Summary of GO synthesis methods.

\begin{tabular}{llllc}
\hline Method & Year & Oxidants & Solvent & Reference \\
\hline Brodie & 1859 & $\mathrm{KClO}_{3}$ & $\mathrm{HNO}_{3}$ & 5 \\
Staudenmaier & 1898 & $\mathrm{KClO}_{3}$ & $\mathrm{HNO}_{3}$ (fuming), & 6 \\
& & & $\mathrm{H}_{2} \mathrm{SO}_{4}$ & 7 \\
Hofmann & 1937 & $\mathrm{KClO}_{3}$ & $\mathrm{HNO}_{3}, \mathrm{H}_{2} \mathrm{SO}_{4}$ & 8 \\
Hummers & 1958 & $\mathrm{NaNO}_{3}, \mathrm{KMnO}_{4}$ & $\mathrm{H}_{2} \mathrm{SO}_{4}$ & 9 \\
Modified Hummers & 1999 & $\mathrm{~K}_{2} \mathrm{~S}_{2} \mathrm{O}_{8}, \mathrm{P}_{2} \mathrm{O}_{5}, \mathrm{KMnO}_{4}$ & $\mathrm{H}_{2} \mathrm{SO}_{4}$ & 10 \\
& 2010 & $\mathrm{KMnO}_{4}$ & $\mathrm{H}_{2} \mathrm{SO}_{4}, \mathrm{H}_{3} \mathrm{PO}_{4}$ & \\
\hline
\end{tabular}

Nevertheless, the synthesis of graphene by these methods is fraught with problems, including defective graphene layers and difficulties in scale-up production. An alternative to mitigate the problem of graphene is to use GO. 
Nowadays, researchers are always interested in finding a filler material which promise to deliver strong and multifunctional properties even with low filler content. In fact, the excellent performance of GO as a filler does not only depend on the inherent properties on itself, but more importantly on the compatibility of GO with the polymer matrices. So, the challenge to achieve a good dispersion and compatibilisation become a major obstacle to these goals. The presence of moieties oxygen functionalities on GO such as carboxyl, epoxide, and hydroxyl leads to lower compatibility with non-polar polymer matrices. Introduction of hydrophobic groups on GO surfaces is an attractive objective especially when aiming for compatibilisation with non-polar material.

In 2006, Stankovich et al. has modified GO with isocyanate to reduce the hydrophilic properties of GO which then can be exfoliated in polar aprotic solvent. ${ }^{11}$ Afterwards, many researchers modified GO with octadecylamine (ODA) through various types of reaction. $\mathrm{Li}$ et al. prepared functionalised GO with ODA through nucleophilic substitution reaction to improve the dispersion of these material with polystyrene matrix. ${ }^{12}$ This method also increase the conductivity of the material where it can be used as a good electrical conductor. $\mathrm{Cao}$, Feng and $\mathrm{Fu}$ prepared functionalised-GO with long alkyl chain by simply using an amidation reaction and the result showed homogenous dispersion and intimate adhesion with polypropylene matrix. ${ }^{13}$ Compton et al. also modified GO with hexylamine followed by reduction processes to improve conductivity of GO which can be an attractive strategy for producing electrically conductive material. ${ }^{14}$ Not only that, Mei et al. modified GO with various alkylamine to tuning hydrophilic properties of GO to hydrophobic. ${ }^{15}$ Based on the literature review, chemicals with long alkyl chains such as ODA have been used for modification of GO not only change the hydrophilic properties of GO to hydrophobic, but also can improve some features such as conductivity, dispersibility and solubility of GO.

Up to now, there are many different method to functionalise GO such as chemical method that usually possesses long reaction time, involving toxic reagents with strictly reaction condition which will limited the large scale production. ${ }^{14,16,17,18}$ Therefore, a novel route that combines the economic benefits and convenience of chemical synthesis with possessing high reduction efficiency, has been an import target for the preparation of functionalised-GO. Recently, gamma-ray $(\gamma$-ray) irradiation has attracted attention among researchers in promising not only environmental friendly method, but also feasible, cost effective and can be perform in room temperature as well. However, the study of $\gamma$-ray radiation induced grafting on surface of GO is still an emerging field. Therefore, in this paper, we demonstrate that GO can be produced using an improved Hummers method without using $\mathrm{NaNO}_{3}$ and proceed with functionalisation with alkylamine throughgamma-irradiation technique. This 
method decreases the cost, time and environmental duty of GO production and functionalisation of them. The effects of different alkyl chain lengths were investigated.

\section{EXPERIMENTAL}

\subsection{Materials}

Graphite powder $(<20 \mu \mathrm{m})$ was obtained from Sigma Aldrich (Missouri, USA). Sulphuric acid $\left(\mathrm{H}_{2} \mathrm{SO}_{4}, 98 \%\right)$ and hydrogen peroxide $\left(\mathrm{H}_{2} \mathrm{O}_{2}, 30 \%\right)$ were obtained from R\&J Chemicals and used as received. Potassium permanganate $\left(\mathrm{KMnO}_{4}, 99.9 \%\right)$ and hydrochloric acid $(\mathrm{HCl}, 36 \%)$ were purchased from SYSTERM. Dodecylamine, tetradecylamine, hexadecylamine and octadecylamine werepurchased from Acros organic. All other chemicals are of analytical grade and used as received without further purification. Distilled water was used for the washings.

\subsection{Preparation of GO}

Graphene oxide was synthesised from graphite using a simplified Hummers' method. $5 \mathrm{~g}$ of graphite were first oxidised by reacting them with concentrated $\mathrm{H}_{2} \mathrm{SO}_{4}(500 \mathrm{ml})$ before adding $\mathrm{KMnO} 4(18 \mathrm{~g})$. The reaction was allowed to go on for 60 hours to fully oxidise graphite to graphite oxide. $\mathrm{H}_{2} \mathrm{O}_{2}$ solution $(5 \% \mathrm{v} / \mathrm{v})$ was added to destroy excess $\mathrm{KMnO}_{4}$ and terminate the oxidation process. The graphite oxide formed was washed several times with $1 \mathrm{M}$ of aqueous $\mathrm{HCl}$ solution to remove $\mathrm{SO}_{4}{ }^{2-}$ ions. Then, the mixture was washed repeatedly with distilled water to remove $\mathrm{Cl}^{-}$ions in the mixture. The washing process was carried out using a simple decantation of the supernatant with a centrifugation technique. The final product was vacuum dried in a vacuum dryer at $80^{\circ} \mathrm{C}$ and pressure of $70 \mathrm{~cm} \mathrm{Hg}$.

\subsection{Functionalisation of GO}

GO (0.6 g) was dissolved and exfoliated in $300 \mathrm{ml}$ deionised water via ultrasonication. The resulting suspension was mixed with the solution of octadecylamine $(0.9 \mathrm{~g})$ in $90 \mathrm{ml}$ ethanol. The mixture was undergo ultrasonication before sending for irradiation treatment. The ${ }^{60} \mathrm{Co}$ gamma ray source was used in room temperature atmosphere with total doses of $25 \mathrm{kGy}$. After irradiation treatment, the resultant product (denoted as GO-A18) was separated by filtration process. The collected solid was thoroughly washed by ethanol several times and dried at $70^{\circ} \mathrm{C}$ in vacuum oven. The dried GO-A18 were ready for characterisations. Similar synthesis steps were followed in preparing 
dodecylamine (GO-A12), tetradecylamine (GO-A14) and hexadecylamine (GOA16) functionalised-GO.

\subsection{Characterisations}

Fourier Transform Infrared spectra were recorded using a spectrum BX Perkin Elmer with UATR technique. The infrared spectra of the samples were recorded in the range of the frequency 280 to $4000 \mathrm{~cm}^{-1}$ at $25^{\circ} \mathrm{C}$. A Perkin Elmer delta series TGA-7 was used for thermogravimetric analysis (TGA) of the samples. About $10-15 \mathrm{mg}$ of each samples were used for the analysis. The samples were heated from $35^{\circ} \mathrm{C}$ to $800^{\circ} \mathrm{C}$ at the heating rate of $10^{\circ} \mathrm{C} / \mathrm{min}$. The analysis was carried out nitrogen at flow rate of $20 \mathrm{ml} / \mathrm{min}$. The weight loss temperature function graph was plotted. XRD with $\mathrm{Cu} \mathrm{K \alpha}$ radiation $(\lambda=1.542 \AA)$ operated at $30 \mathrm{kV}$ and $30 \mathrm{~mA}$ was used to determine the interlaying spacing of the GO sheets before and after functionalised. Data were collected within the range of scattering angles $(2 \theta)$ of $2^{\circ}$ to $30^{\circ}$ at the rate of $2^{\circ} / \mathrm{min}$. ${ }^{1} \mathrm{H}$ NMR spectra were obtained from JOEL JMTC spectrometer $(500 \mathrm{MHz})$. Chemical shift were recorded in ppm relatives to tetramethylsilane (TMS, $0.00 \mathrm{ppm}$ ) signal and deuterated chloroform $\left(\mathrm{CD}_{3} \mathrm{Cl}\right)$ was used as a solvent. X-ray photoelectron spectroscopy (XPS) was employed to investigate the surface chemical state of the GO and functionalised-GO. The analysis was performed on a ULVAC-PHI Quantera II (Ulvac-PHI, INC) instrument using a monochromatic Al-K $\alpha$ (1486.6 $\mathrm{eV}$ ) source at power of $25.6 \mathrm{~W}$. Scanning electron microscopy (SEM) images were performed at magnification of $1000 \times$ by JEOL JSM-6400F.

\section{RESULTS AND DISCUSSION}

Natural graphite was used as the starting material. The inter-graphene layers can be intercalated by various molecular species or ions, during which the interlayer spacing changed. We employed concentrated sulphuric acid to oxidise the natural graphite powders. The yields (the weight of GO divided by weight of graphite powder) of GO produced was measure to be $\sim 100 \%$. This indicates that the solution of concentrated $\mathrm{H}_{2} \mathrm{SO}_{4}$ containing $\mathrm{KMnO}_{4}$ is capable of oxidising graphite to $\mathrm{GO}$ with high product yield, even without the assistance of $\mathrm{NaNO}_{3}$ as used in Hummers method.

\subsection{X-ray Diffraction (XRD)}

The mechanism of GO producing is mainly the generation of oxygen containing groups on graphene's surface when oxidation process take place. During oxidation process, hydroxyl, carbonyl, epoxy or peroxy groups were bonded to the edges of basal planes of the graphite structure. Simultaneously, 
carbon hydrolysation occurred and the $\mathrm{sp}^{2}$ bonds changed to $\mathrm{sp}^{3}$ bonds. At the same time, $\mathrm{H}_{2} \mathrm{O}$ or $\mathrm{SO}_{4}{ }^{2-}$ ions could insert themselves into the graphene layer to increase the interlayer spacing. In Figure 1(a) of XRD results, there is only one diffraction peak around $2 \theta=26.4^{\circ}$ in pristine graphite, a typical reflection peak (002) in graphite, and the d-spacing is about $0.334 \mathrm{~nm}$. As oxidation proceeds, the intensity of this peak gradually weakened and finally disappeared. Simultaneously, the diffraction peak at about $10.2^{\circ}$ appeared correspond to an increasing the interlayer spacing from $0.334 \mathrm{~nm}$ of graphite to $0.824 \mathrm{~nm}$ of GO. The interlayer spacing of graphite oxide is increased due to the present of abundant covalently bounded oxygen-containing functional group on the graphene sheets during oxidation process. These abundant oxygen containing functional groups weaken the van de Waals interactions between graphene sheets and thus increase the distance between graphene sheets. ${ }^{19,20,21,22}$ The oxidation process itself also causes of the breaking of the graphite structure into smaller fragments. ${ }^{23}$ Upon functionalisation with alkylamine, the interlayer spacing was further enlarged by intercalation of the long chain alkylamine between the graphene layers. XRD patterns of $\mathrm{GO}$ and various type of alkylamine functionalised-GO (GO-A8, GO-A12, GO-A18) were shown in Figure 1(b). The interaction of alkylamine with GO may decrease the order of crystallinity in graphite or deteriorate the degree of graphite crystallinity, which induces the main diffraction peak to become broader and slightly shifting to low angle. ${ }^{24}$ After functionalisation, the peak was further shifted to $3.8^{\circ}$ (GO-A12), $3.5^{\circ}$ (GO$\mathrm{A} 14$ ), and $3.3^{\circ}$ (GO-A16 and GO-A18) correspond to the expansion on the interlayer of GO sheets from $0.824 \mathrm{~nm}$ to $2.325 \mathrm{~nm}$ (GO-A12), $2.525 \mathrm{~nm}$ (GOA14) and $2.677 \mathrm{~nm}$ (GO-A16 and GO-A18) respectively. On the other hand, the broad peak around $18^{\circ}-24^{\circ}$ was arise due to the formation of reaggregated graphene sheets. ${ }^{18}$ This change supports the successful functionalisation of alkylamine to GO through $\gamma$-ray irradiation technique. 

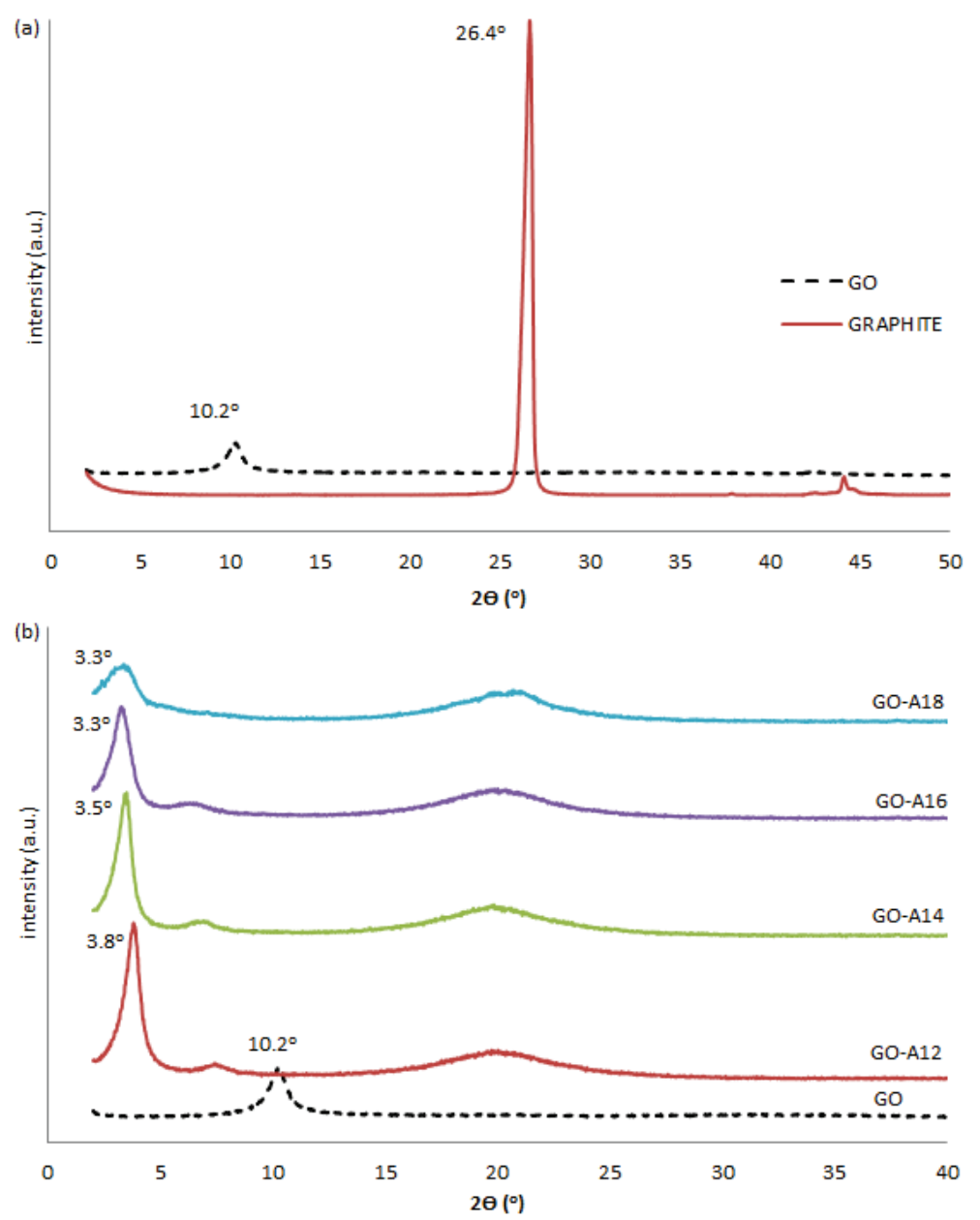

Figure 1: XRD spectra of (a) GO and graphite (b) GO and functionalised-GOs.

\subsection{Fourier Transform Infrared (FTIR)}

FTIR analysis can be one of the direct evidence for GO's functionalisation as it provides information about functional groups that present in the sample. FTIR spectra of GOand functionalised-GO were presented in Figure 2(a). Generally, GO shows typical peaks of broad band at $\sim 3340 \mathrm{~cm}^{-1}$ which associated to hydroxyl groups, while $1717 \mathrm{~cm}^{-1}$ correspond to carboxyl groups, $1625 \mathrm{~cm}^{-1}$ is $\mathrm{C}=\mathrm{C}$ in aromatic ring and $1060 \mathrm{~cm}^{-1}$ is for $\mathrm{C}-\mathrm{O}-\mathrm{C}$ in epoxide respectively. ${ }^{20,21,24}$ Thus the FTIR data verify the existence of oxygen containing functional groups in GO. Upon functionalisation with various alkylamine through $\gamma$-ray irradiation, the peaks around $2852-2920 \mathrm{~cm}^{-1}$ appeared corresponding to the $\mathrm{C}-\mathrm{H}$ stretching vibration of the alkyl chain $\left(\mathrm{CH}_{2}\right)$ together 
with peak around $\sim 728 \mathrm{~cm}^{-1}$. The peak at $1590 \mathrm{~cm}^{-1}$ of functionalised-GO is probably due to the $\mathrm{N}-\mathrm{H}$ bend, while the same peak appears at $1585 \mathrm{~cm}^{-1}$ in pristine ODA in Figure 2(b). The peak at $1082 \mathrm{~cm}^{-1}$ in functionalised-GO is probably due to the shift of $\mathrm{C}-\mathrm{N}$ stretch at $1067 \mathrm{~cm}^{-1}$ in parent amine ODA. The peak at $1486 \mathrm{~cm}^{-1}$ of ODA belongs to $\mathrm{C}-\mathrm{H}$ bend, and it moves to $1464 \mathrm{~cm}^{-1}$ in functionalised-GO. The spectral shift observed might result from the functionalised alkylamine with GO. The band at 1717 and $1060 \mathrm{~cm}^{-1}$ corresponding to carboxyl and epoxide were weakened after functionalised and a new peak at $1082 \mathrm{~cm}^{-1}(\mathrm{C}-\mathrm{N})$ appears, indicating the formation of $\mathrm{C}-\mathrm{NH}-\mathrm{C}$ bands. ${ }^{17}$ Generally, $\gamma$-ray irradiation method illustrating the simultaneous functionalisation and slightly reduction on $\mathrm{GO}^{25,26,27,28} \mathrm{~A}$ certain decrease in the intensity of the bands at $\sim 1717,1625$ and $1060 \mathrm{~cm}^{-1}$ could be related to the evolution of water or labile oxygen chemical groups during $\gamma$-ray irradiation as reported by Anson et al. ${ }^{29}$
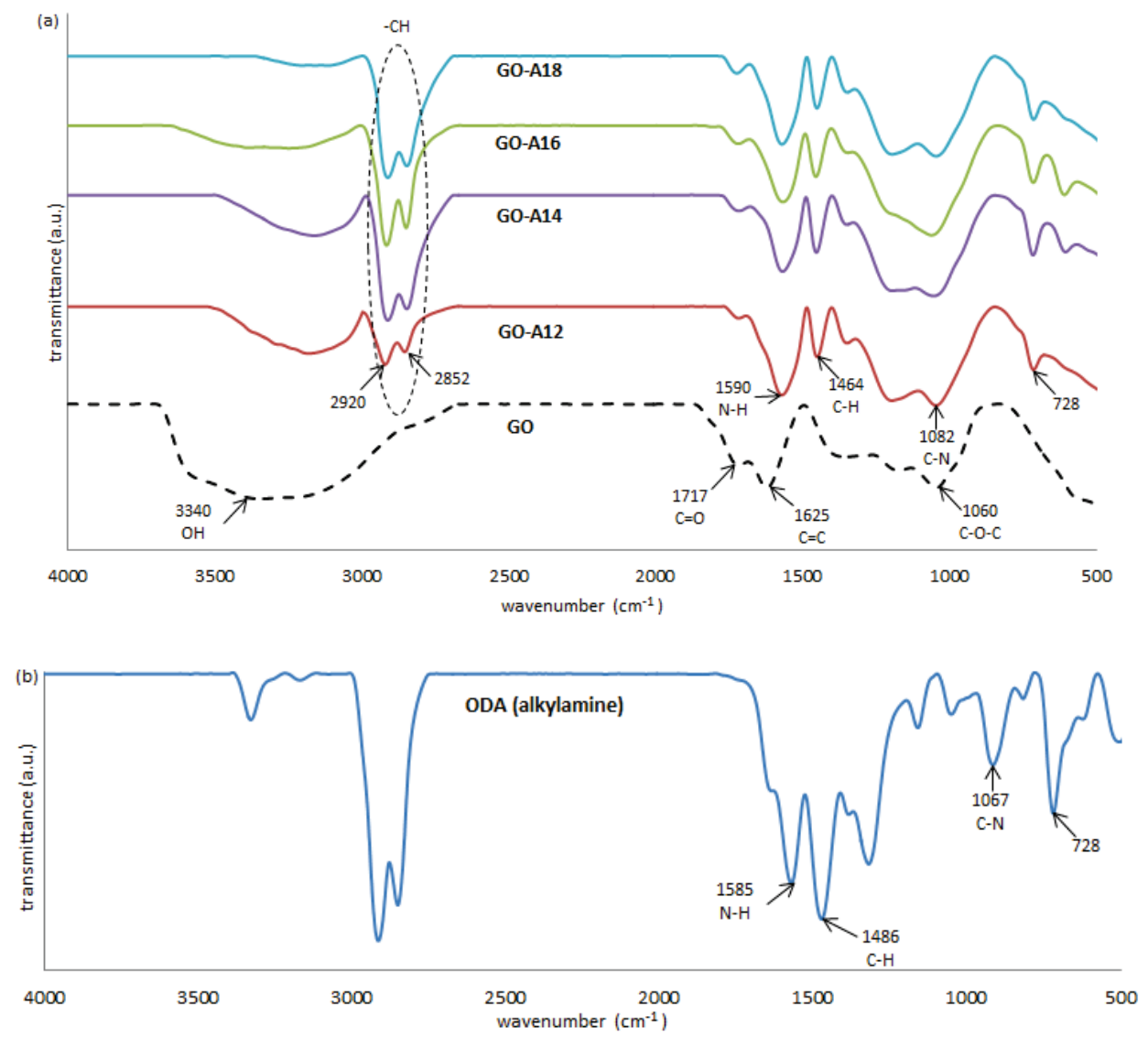

Figure 2: FTIR spectra of (a) GO and functionalised-GOs and (b) ODA (octadecylamine). 


\subsection{Thermogravimetric Analysis (TGA)}

TGA was used to investigate thermal stability of the samples. Figure 3(a) depicts the TGA of GO and functionalised-GOs. Pristine GO showed a major weight loss of about $80 \%$ in temperature range of $130^{\circ} \mathrm{C}-200^{\circ} \mathrm{C}$ corresponding to the decomposition of labile oxygen-containing functionalities and illustrating lower thermal stability. A significant drop of GO in mass around $300^{\circ} \mathrm{C}$ is due to more stable oxygen-containing functional groups and the bulk pyrolysis of carbon skeleton. ${ }^{30}$ After functionalisation with different chain length of amine through $\gamma$-ray irradiation, the result showed no significant weight loss up to $175^{\circ} \mathrm{C}$. This showed an improvement in thermal stability and decelerated degradation rate, which is possibility due to interaction of grafted alkylamine and GO surfaces. ${ }^{17}$ The thermal stability of functionalised-GO increased substantially below $400^{\circ} \mathrm{C}$ with increasing chain length of grafted alkylamine. Figure 3(b) showed DTG thermograms of the samples. The major weight loss is about $\sim 60 \%$ in therange of $178^{\circ} \mathrm{C}-530^{\circ} \mathrm{C}$ was attributed to the thermal decomposition of alkylamine chain. Interestingly, below $100^{\circ} \mathrm{C}$ of functionalised-GO showed less than $10 \%$ weight loss which indicating an enhanced hydrophobicity that minimised the amount of adsorbed water ${ }^{18}$ compared to pristine GO which usually exhibited a weight loss about $\sim 18 \%$ below $100^{\circ} \mathrm{C}$ corresponding to evaporation of the trapped water molecules in the GO. ${ }^{31}$ After functionalisation, results showed small degradation peak in range $200^{\circ} \mathrm{C}$ contributed to the loss of small fragment of alkylamine. However, when the chain length of alkylamine grafted was increased (GO-A12 and GO-A18), two degradation temperature were observed. The second degradation peak arise around $350^{\circ} \mathrm{C}$ was supported by degradation of grafted long alkylamine that intercalated between GO sheets. ${ }^{18}$

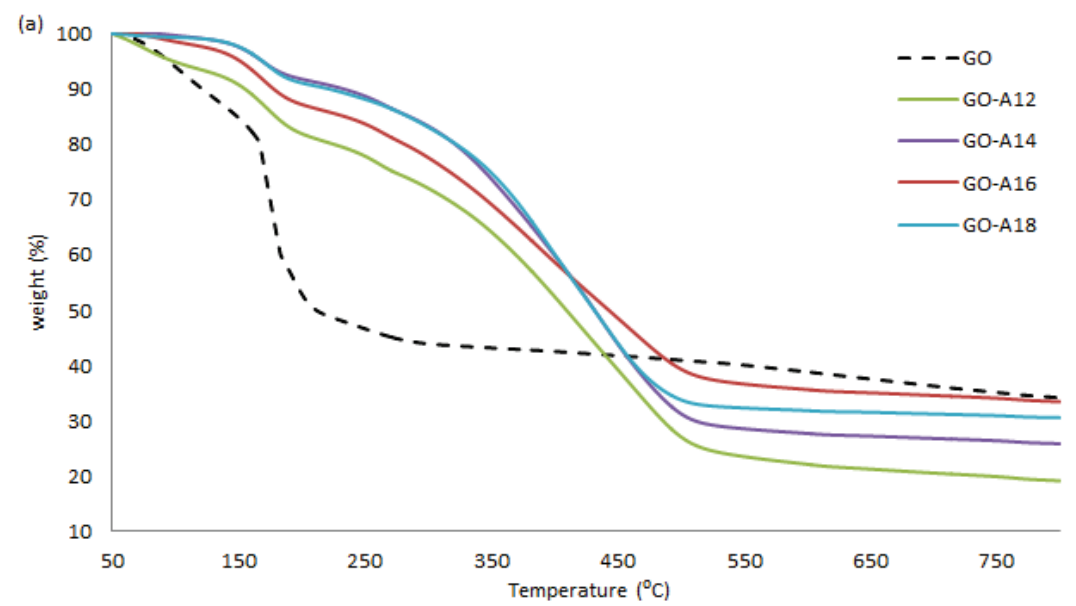

Figure 3: (a) TGA and (b) DTG thermograms of GO and functionalised-GOs. (continued on next page) 
(b)

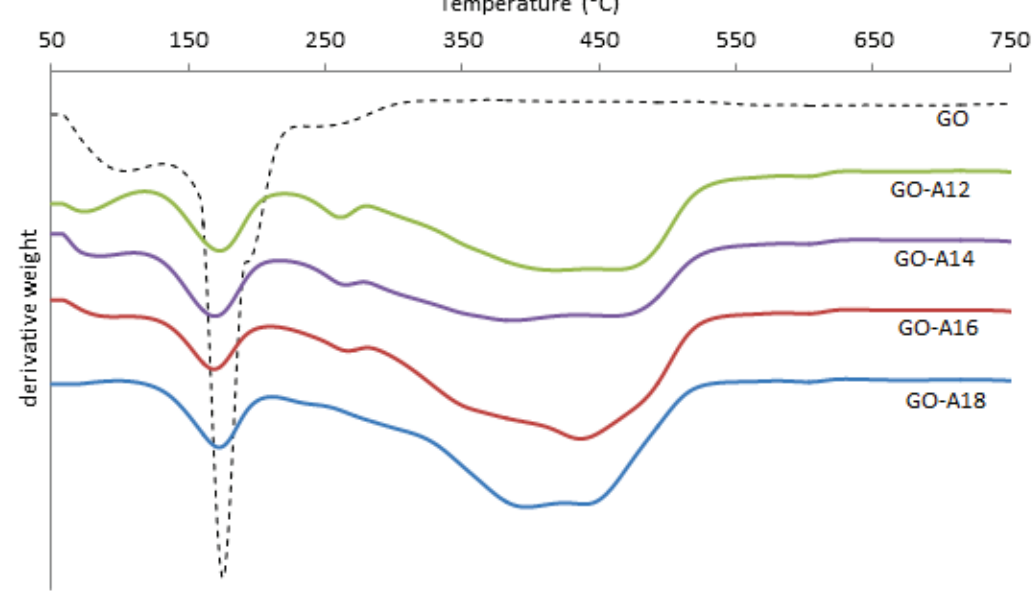

Figure 3: (continued).

\subsection{Nuclear Magnetic Resonance (NMR)}

NMR was used to elucidate the bonding chemistry between alkylamine and GO after functionalisation and the result was presented on Figure 4. The peak at $\sim 7 \mathrm{ppm}$ is assigned to $\mathrm{CDCl}_{3}$ solvent. ${ }^{1} \mathrm{H}$ NMR spectrum shows the presence of alkylamine chain on sample by appearing of several signals at $0.88\left(-\mathrm{CH}_{3}\right.$, small intensity) and $1.25 \mathrm{ppm}\left(-\mathrm{CH}_{2}\right.$, very intense). It also showed small peak around 3.14 and $3.28 \mathrm{ppm}$ which corresponding to $\mathrm{H}$ peak of $(-\mathrm{NH})$ which supports the reaction of alkylamine with GO. ${ }^{12,17,18}$

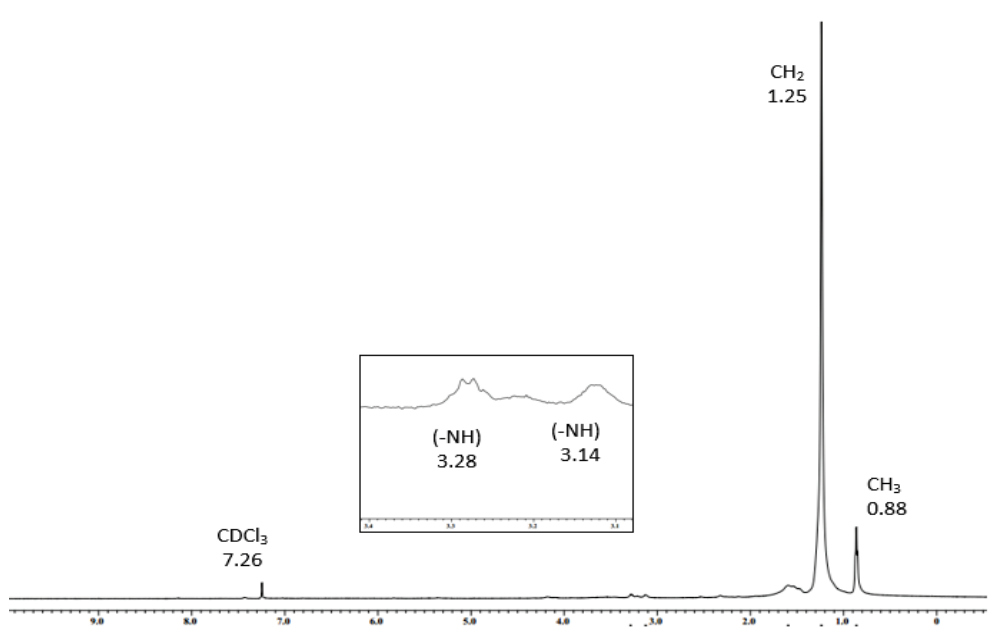

Figure 4: ${ }^{1} \mathrm{H}$ NMR spectrum of GO-A18 in $\mathrm{CDCl}_{3}$. 


\subsection{X-ray Photoelectron Spectroscopy}

Additionally, the successful of reduction and functionalisation is also proved by the XPS analysis and the results are displayed in Figure 5 (a-d). Survey scan results of the GO showed two strong peak at $280 \mathrm{eV}$ and $532 \mathrm{eV}$, which correspond to $\mathrm{C} 1 \mathrm{~s}$ and $\mathrm{O} 1 \mathrm{~s}$ peaks (Figure 5[a]). Functionalisation of long alkylamine on the GO surface resulted in a significant increase in peak intensity of the $\mathrm{C} 1 \mathrm{~s}$ peak $(280 \mathrm{eV})$ with extremely attenuated in $\mathrm{O} 1 \mathrm{~s}$ peak $(532 \mathrm{eV})$ which also be found by Zhang et al. ${ }^{32}$ Functionalisation also corroborated by the decrease in the $\mathrm{O} / \mathrm{C}$ ratio from 2.34 (GO) to 0.30 (GO-A18) as showed in Table 2. Successful of functionalisation is further revealed by the appearance of a new peak at $400 \mathrm{eV}$ (N1s) (Figure 5[a]), which is further confirmed from the rise in the N/O ratio (Table 1). Deconvolution of high resolution spectra $(\mathrm{C} 1 \mathrm{~s}, \mathrm{O} 1 \mathrm{~s}$ and N1s) was carried out and a few representative results are included in Figure 5 (b, $\mathrm{c}, \mathrm{d}$ and e). The resolution of XPS spectrometer does not allow to analyse peaks of $\mathrm{C}-\mathrm{C}$ and $\mathrm{C}=\mathrm{C}$ separately, therefore it will be treated as a single signal and compared with peaks corresponding to the carbon atoms bounded with other groups. Deconvolution of $\mathrm{C} 1 \mathrm{~s}$ spectra of pristine $\mathrm{GO}$ showed four major peaks that correspond to $\mathrm{C}-\mathrm{C} / \mathrm{C}-\mathrm{H}(284.79 \mathrm{eV}), \mathrm{C}-\mathrm{O}(287.00 \mathrm{eV}), \mathrm{C}=\mathrm{O}(288.62 \mathrm{eV})$ and $\mathrm{COOH}(290.51 \mathrm{eV})$. This indicates considerably degree of oxidation of GO that means the presence of different oxygen functional groups in GO structure. ${ }^{33}$ In addition, functionalisation of GO with alkylamine such as octadecylamine (GO-A18) resulted in significant rise in $\mathrm{C}-\mathrm{C} / \mathrm{C}-\mathrm{H}$ and drastic decrease in the peaks corresponding to $\mathrm{C}-\mathrm{O}, \mathrm{C}=\mathrm{O}$ and $\mathrm{COOH}$, along with the appearance of peak at $285.79 \mathrm{eV}(\mathrm{C}-\mathrm{N}){ }^{34}$ Information provided by the analysis of the $\mathrm{O} 1 \mathrm{~s}$ complements the information provided by the analysis of the C1s spectra. By reference to the changes of $\mathrm{C} 1 \mathrm{~s}$ peak upon functionalisation, there is sudden decrease of $\mathrm{C}-\mathrm{O}$ and $\mathrm{C}=\mathrm{O}$ hence only two peaks appeared in the deconvoluted $\mathrm{O} 1 \mathrm{~s}$ spectra. This fact is further revealed from the deconvoluted N1s spectra, which showed two peaks at $399.49 \mathrm{eV}(\mathrm{C}-\mathrm{N})$ and at $400.50 \mathrm{eV}(\mathrm{N}-\mathrm{H})$.

Table 2: Survey scan results for functionalised-GOs samples.

\begin{tabular}{lcc}
\hline Sample & O/C ratio & N/O ratio \\
\hline GO & 2.34 & 0.08 \\
GO-A12 & 0.51 & 0.91 \\
GO-A14 & 0.48 & 0.74 \\
GO-A16 & 0.36 & 0.74 \\
GO-A18 & 0.30 & 1.55 \\
\hline
\end{tabular}



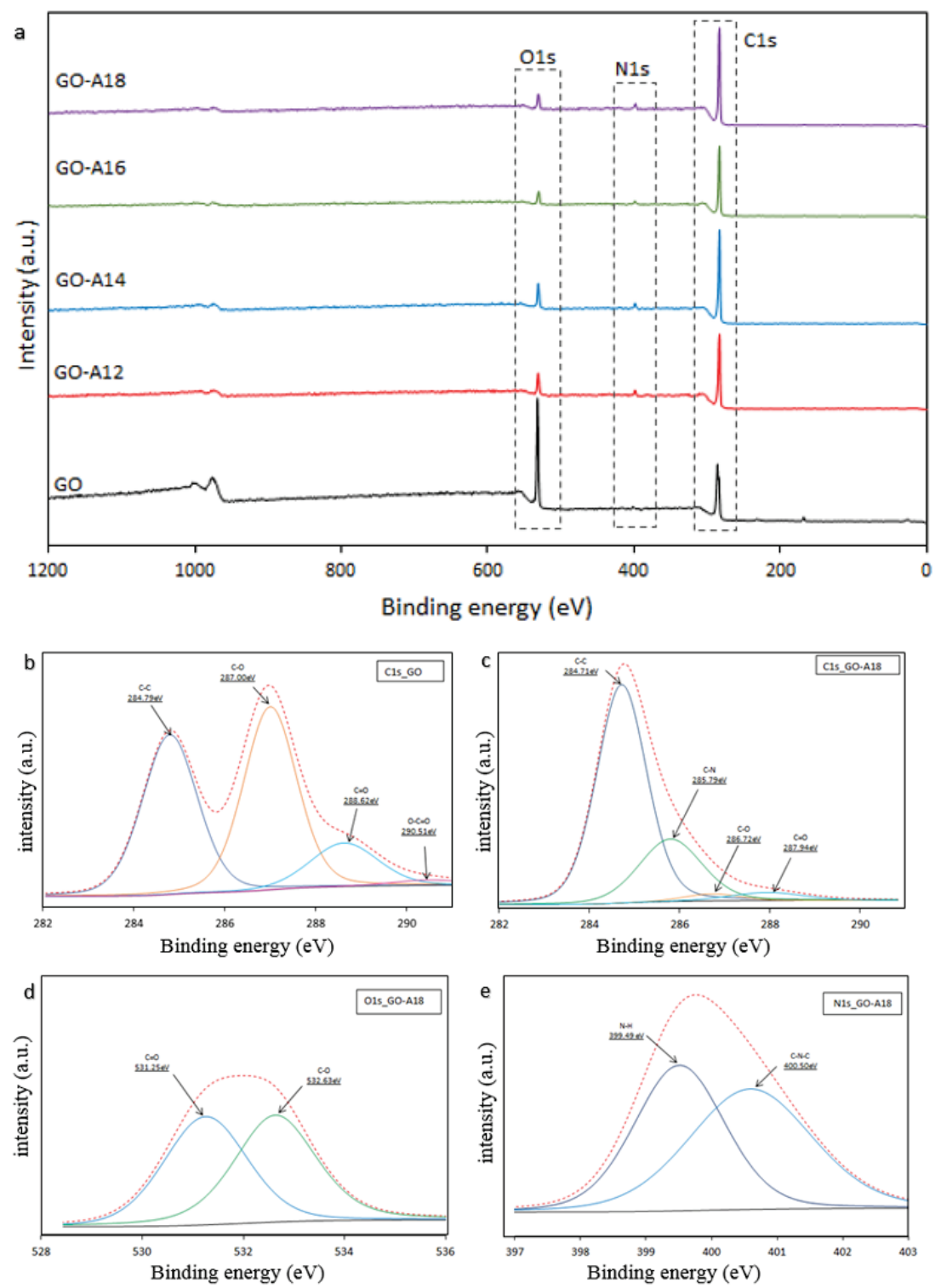

Figure 5: Binding energy and XPS results of GO and functionalised-GOs.

\subsection{Scanning Electron Microscopy (SEM)}

Surface morphologies of the pristine GO and functionalised-GO were determined by using SEM and the micrographs were shown on Figure 6. Pristine GO showed close stacking of sheets with relatively smooth surface together with 
very sharp edges ${ }^{35}$ compared to functionalised-GO. However, samples show crumpled flakes with wrinkles and some folded regions in random orientation after functionalisation with alkylamine. For GO-A12 there are only a few aggregated domains that are less dense and the surface roughness is quite small. Increasing the chain length of alkylamine (GO-A14, GO-A16 and GO-A18) resulted in the formation of many large and more obvious thick domain with high surface roughness indicated that this long-chain of alkylamine were successfully attached on the GO surfaces. The difference in surface roughness between GO and GO-A can be explained in terms of their chemical properties. GO is hydrophilic which can easily dispersed in water to form individual-sheets with smooth surface due to the slow vacuum-filtration process. However, an attachment of long alkyl chain onto GO changed them to hydrophobic leading to a poor dispersion in polar solvent and tend to form a large aggregated domains due to a fast vacuum-filtration process. This special morphological features not only enhances the surfaces roughness, but substantially tuning the wettability properties of solid surfaces and dispersion behaviours of the samples. ${ }^{35,36,37}$

\section{CONCLUSION}

This work suggests that pristine graphite, when treated directly by appropriate chemical can readily generate exfoliated graphene oxide without the need for any chemical agents. This method does not generate toxic gas, in contrast to Hummers' method. With further surface modifications, GO with multi properties can be accessible, thereby further expecting in many application of GO. Besides, GO was successfully functionalised and slightly reduced by gamma-ray irradiation in ethanol/water blend solution. The chemical structural changes due to the radiation induced functionalisation are proved by means of XRD, FTIR, NMR, XPS and TG analysis. The elimination of those hydroxyl groups and the decarboxylation effect is demonstrated. Alkyl groups are attached onto GO sheets due to the recombination of radicals. The SEM images show that functionalised-GO obtained having high surface roughness. All the results indicate that the radiation induced functionalisation in ethanol/water is a good method to obtain functionalised-GO which is desirable for hydrophobic materials preparation. 


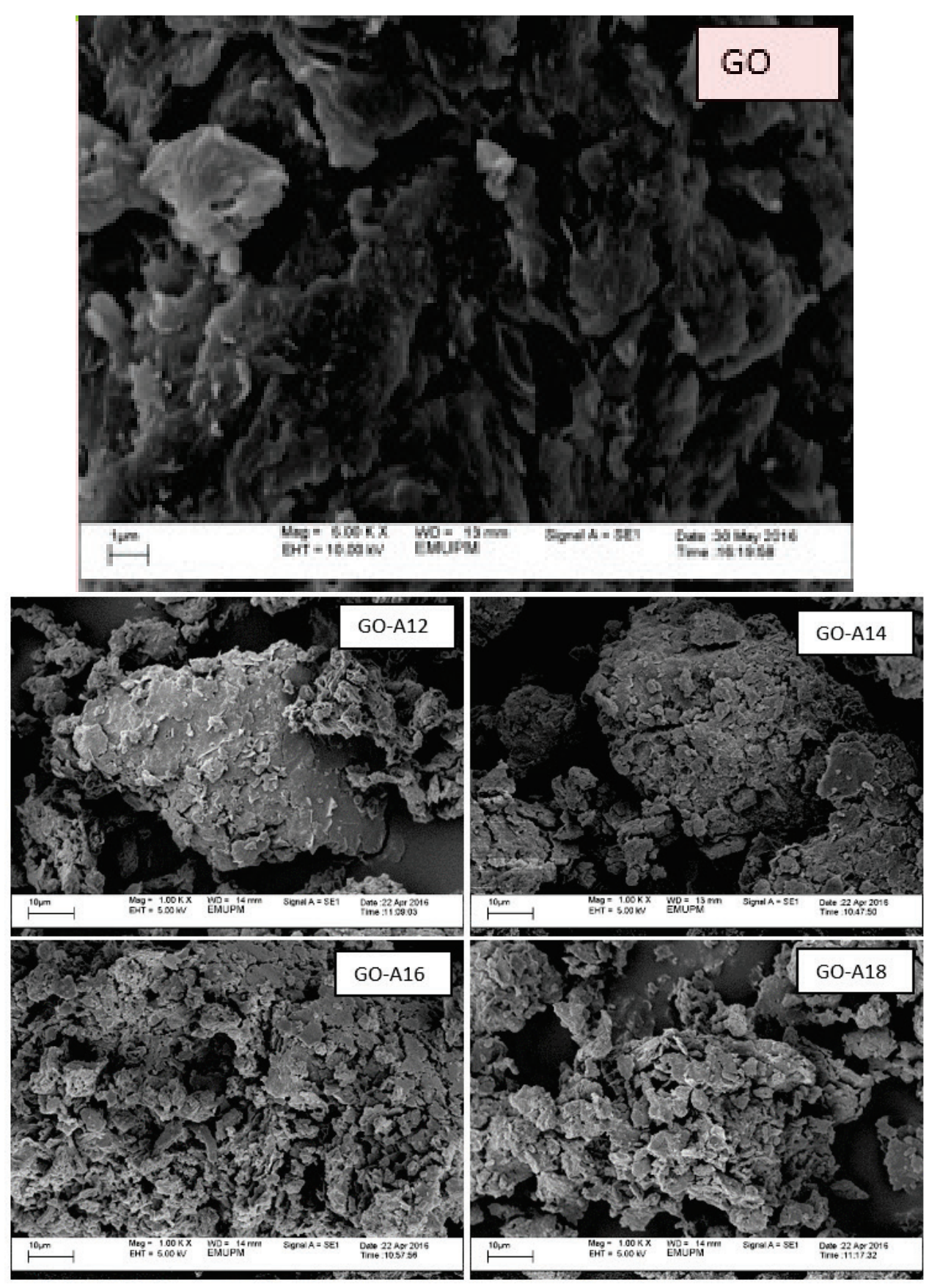

Figure 6: SEM micrography of GO and functionalised-GOs. 


\section{REFERENCES}

1. Hernandez, Y. et al. (2008). High-yield production of graphene by liquidphase exfoliation of graphite. Nat. Nano., 3(9), 563-568, https://doi.org/10.1038/nnano.2008.215.

2. Choucair, M., Thordarson, P. \& Stride, J. A. (2009). Gram-scale production of graphene based on solvothermal synthesis and sonication. Nat. Nano., 4(1), 30-33, https://doi.org/10.1038/nnano.2008.365.

3. Lerf, A. et al. (1998). Structure of graphite oxide revisited. The J. Phys. Chem. B, 102(23), 4477-4482, https://doi.org/10.1021/jp9731821.

4. Park, S. \& Ruoff, R. S. (2009). Chemical methods for the production of graphenes. Nat. Nano., 4(4), 217-224, https://doi.org/10.1038/nnano. 2009.58.

5. Brodie, B. C. (1859). On the atomic weight of graphite. Phil. Trans. R. Soc. Lond., 149, 249-259, https://doi.org/10.1098/rstl.1859.0013.

6. Staudenmaier, L. (1898). A process for presentation of graphitic acid. $J$. Am. Chem. Soc., 31, 1481-1487.

7. Hofmann, U. \& König, E. (1937). Investigations on graphitic. J. Inorg. Gen. Chem., 234, 311-336.

8. Hummers Jr, W. S. \& Offeman, R. E. (1958). Preparation of graphitic oxide. J. Am. Chem. Soc., 80(6), 1339-1339, https://doi.org/10.1021/ ja01539a017.

9. Kovtyukhova, N. I. et al. (1999). Layer-by-layer assembly of ultrathin composite films from micron-sized graphite oxide sheets and polycations. Chem. Mater., 11(3), 771-778, https://doi.org/10.1021/ cm981085u.

10. Marcano, D. C. et al. (2010). Improved synthesis of graphene oxide. ACS Nano, 4(8), 4806-4814, https://doi.org/10.1021/nn1006368.

11. Stankovich, S. et al. (2006). Synthesis and exfoliation of isocyanatetreated graphene oxide nanoplatelets. Carbon, 44(15), 3342-3347, https://doi.org/10.1016/j.carbon.2006.06.004.

12. Li, W. et al. (2011). Simultaneous surface functionalization and reduction of graphene oxide with octadecylamine for electrically conductive polystyrene composites. Carbon, 49(14), 4724-4730, https://doi.org/10.1016/j.carbon.2011.06.077.

13. Cao, Y., Feng, J. \& Wu, P. (2010). Alkyl-functionalized graphene nanosheets with improved lipophilicity. Carbon, 48(5), 1683-1685, https://doi.org/10.1016/j.carbon.2009.12.061.

14. Compton, O. C. et al. (2010). Electrically conductive "alkylated" graphene paper via chemical reduction of amine-functionalized graphene oxide paper. Adv. Mater., 22(8), 892-896, https://doi.org/10. 1002/adma.200902069. 
15. Mei, Q. et al. (2010). Highly efficient photoluminescent graphene oxide with tunable surface properties. Chem. Comm., 46(39), 7319-7321, https://doi.org/10.1039/c0cc02374d.

16. Guan, W. et al. (2013). Amine modified graphene as reversed-dispersive solid phase extraction materials combined with liquid chromatography: Tandem mass spectrometry for pesticide multi-residue analysis in oil crops. J. Chromatogr. A., 1286, 1-8, https://doi.org/10.1016/j.chroma. 2013.02.043.

17. Zhang, S. P. \& Song, H. O. (2012). Supramolecular graphene oxidealkylamine hybrid materials: variation of dispersibility and improvement of thermal stability. New J. Chem., 36(9), 1733-1738, https://doi.org/ 10.1039/c2nj40214a.

18. Shanmugharaj, A. M. et al. (2013). Synthesis, characterization, and surface wettability properties of amine functionalized graphene oxide films with varying amine chain lengths. J. Colloid Interface Sci., 401, 148-154, https://doi.org/10.1016/j.jcis.2013.02.054.

19. Gao, W. (2015). The chemistry of graphene oxide. In Graphene oxide. Cham, Switzerland: Springer International Publishing, 61-95, https://doi.org/10.1007/978-3-319-15500-5_3.

20. Chen, J. et al. (2013). An improved Hummers method for eco-friendly synthesis of graphene oxide. Carbon, 64, 225-229, https://doi.org/10. 1016/j.carbon.2013.07.055.

21. Chen, J. et al. (2015). High-yield preparation of graphene oxide from small graphite flakes via an improved Hummers method with a simple purification process. Carbon, 81, 826-834, https://doi.org/10. 1016/j.carbon.2014.10.033.

22. Wang, C. et al. (2016). Preparation and properties of octadecylamine modified graphene oxide/styrene-butadiene rubber composites through an improved melt compounding method. J. Appl. Polym. Sci., 133(4), 42907, https://doi.org/10.1002/app.42907.

23. Li, Z. et al. (2009). How graphene is cut upon oxidation? J. Am. Chem. Soc., 131(18), 6320-6321, https://doi.org/10.1021/ja8094729.

24. Chen, Y. et al. (2015). Superior performance nanocomposites from uniformly dispersed octadecylamine functionalized multi-walled carbon nanotubes. $C, 1(1), 58-76$, https://doi.org/10.3390/c1010058.

25. Guerrero-Contreras, J. \& Caballero-Briones, F. (2015). Graphene oxide powders with different oxidation degree, prepared by synthesis variations of the Hummers method. Mater. Chem. Phys., 153, 209-220, https://doi.org/10.1016/j.matchemphys.2015.01.005. 
26. Dumée, L. F. et al. (2014). Tuning the grade of graphene: Gamma ray irradiation of free-standing graphene oxide films in gaseous phase. Appl. Surf. Sci., 322, 126-135, https://doi.org/10.1016/j.apsusc.2014.10.070.

27. Dumée, L. F. et al. (2014). Single step preparation of meso-porous and reduced graphene oxide by gamma-ray irradiation in gaseous phase. Carbon, 70, 313-318, https://doi.org/10.1016/j.carbon.2013.12.094.

28. Li, J. et al. (2014). $\gamma$-ray irradiation effects on graphene oxide in an ethylenediamine aqueous solution. Radiat. Phys. Chem., 94, 80-83, https://doi.org/10.1016/j.radphyschem.2013.06.029.

29. Ansón-Casaos, A. et al. (2014). The effect of gamma-irradiation on fewlayered graphene materials. Applied Surface Science, 301, 264-272, https://doi.org/10.1016/j.apsusc.2014.02.057.

30. Zhu, C. et al. (2010). Reducing sugar: New functional molecules for the green synthesis of graphene nanosheets. ACS Nano, 4(4), 2429-2437, https://doi.org/10.1021/nn1002387.

31. Choudhary, S., Mungse, H. P. \& Khatri, O. P. (2012). Dispersion of alkylated graphene in organic solvents and its potential for lubrication applications. J. Mater. Chem., 22(39), 21032-21039, https://doi.org/ 10.1039/c2jm34741e.

32. Zhang, B. et al. (2012). Radiation induced reduction: An effective and clean route to synthesize functionalized graphene. J. Mater. Chem., 22(16), 7775-7781, https://doi.org/10.1039/c2jm16722k.

33. Sobon, G. et al. (2012). Graphene oxide vs. reduced graphene oxide as saturable absorbers for Er-doped passively mode-locked fiber laser. Opt. Express, 20(17), 19463-19473, https://doi.org/10.1364/OE.20.019463.

34. Ryu, S. H. \& Shanmugharaj, A. M. (2014). Influence of long-chain alkylamine-modified graphene oxide on the crystallization, mechanical and electrical properties of isotactic polypropylene nanocomposites. Chemical Engineering Journal, 244, 552-560, https://doi.org/10. 1016/j.cej.2014.01.101.

35. Ma, W. S. et al. (2013). Preparation and characterization of long-chain alkyl silane-functionalized graphene film. J. Mater. Sci., 48(1), 156-161, https://doi.org/10.1007/s10853-012-6723-5.

36. Bao, H. et al. (2011). Chitosan-functionalized graphene oxide as a nanocarrier for drug and gene delivery. Small, 7(11), 1569-1578, https://doi.org/10.1002/smll.201100191.

37. Carreira, L. G. et al. (2013). Organically-expanded graphite/octadecylamine: Structural, thermal and relaxation evaluation. Mater. Sci. Appl., 4, 281-286, https://doi.org/10.4236/msa.2013.45035. 